\title{
Triggering Star Formation by Galaxy-Galaxy Interactions
}

\author{
Tissera, P. B. \\ Institute of Astronomy and Space Science, Conicet, Argentina \\ Alonso, M. S. \\ Complejo Astronómico El Leoncito, Conicet, Argentina \\ Lambas, D. G., Coldwell, G.
}

IATE, Observatorio Astronómico Córdoba, Conicet, Argentina

\begin{abstract}
We analyzed the effects of having a close companion on the star formation activity of galaxies in the $8 \mathrm{~K}$ galaxy pair catalog selected from the 2dFGRS. We found that, statistically, galaxies with $r_{\mathrm{p}}<25 \mathrm{~h}^{-1}$ $\mathrm{kpc}$ and $\Delta V<100 \mathrm{~km} \mathrm{~s}^{-1}$ have enhanced star formation with respect to isolated galaxies with the same luminosity and redshift distribution. Our results suggest that the physical processes at work during tidal interactions can overcome the effects of environment, except in dense regions.
\end{abstract}

Different physical processes are thought to be involved in the regulation of star formation in galaxies, but it is now accepted that mergers and interactions play an important role as different observational and numerical works have shown. However, only recently it has been possible to study the effects of interactions on a statistical basis. Barton et al. (2000) found that the star formation activity correlates with proximity in projected distance and velocity difference by analyzing a sample of approximately 200 pairs. The release of the 2dFGRS opened the possibility of carrying out an analysis of star formation in galaxy pairs on a statistical basis. By cross-correlating the galaxy pair catalog with the group catalog of Merchán \& Zandivarez (2003, in preparation) galaxy pairs where classified according to the environment. In this work we focus on the question of how close galaxies have to be in order to show star formation activity enhancement. For this purpose, we will always compare the properties of galaxies in pairs with those of isolated galaxies selected from the $2 \mathrm{dF}$ with the same redshift and luminosity distribution.

We estimated the birth rate parameter $b=S F R /<S F R>$ for galaxies in the 2dFGRS from the correlation between the $\eta$ spectral type and the $H_{\alpha}$ equivalent width (see Lambas et al. 2003, for details). Note that the SFRs are deduced from $H_{\alpha}$ and no dust effects have been considered. We calculated the mean $b$ parameter for the neighbors within concentric spheres centered at a given galaxy. The center galaxies were chosen according to their spectral parameters $\eta$ (see Fig. 1a). A similar calculation was performed by binning in relative velocity separation. From this analysis we found that $b$ in galaxies in pairs satisfying $r_{\mathrm{p}}<100 \mathrm{~h}^{-1} \mathrm{kpc}$ and $\Delta V<350 \mathrm{~km} \mathrm{~s}^{-1}$ is enhanced. By applying 
these two criteria, we selected approximately 9000 pairs with $z<0.1$ in high and low density environments. We also constructed a galaxy control sample by identifying galaxies without a close companion in the field (or cluster) with the same redshift and luminosity distribution as galaxies in pairs. We then estimated the mean $b$ parameters for galaxies in pairs in projected and relative velocity bins, and the mean $b$ value of the control sample. By equating them, we found that the star formation activity is statistically enhanced by the presence of a companion from $r_{\mathrm{p}} \approx 25 \mathrm{~h}^{-1} \mathrm{kpc}$ and $\Delta V<100 \mathrm{~km} \mathrm{~s}^{-1}$ in comparison to isolated galaxies (see Fig. 1b). This analysis, extended to galaxy pairs in groups (Alonso et al. 2004), yields similar results, except for galaxy pairs in very high density regions where both the colors and $b$ show no star formation activity in the present. Hence, statistically, tidal fields seem to be efficient in enhancing the star formation activity for very close pairs. The relative velocity and projected separation thresholds are independent of environment, suggesting that galaxygalaxy interactions can be a main, ubiquitous motor of star formation activity in the Universe.
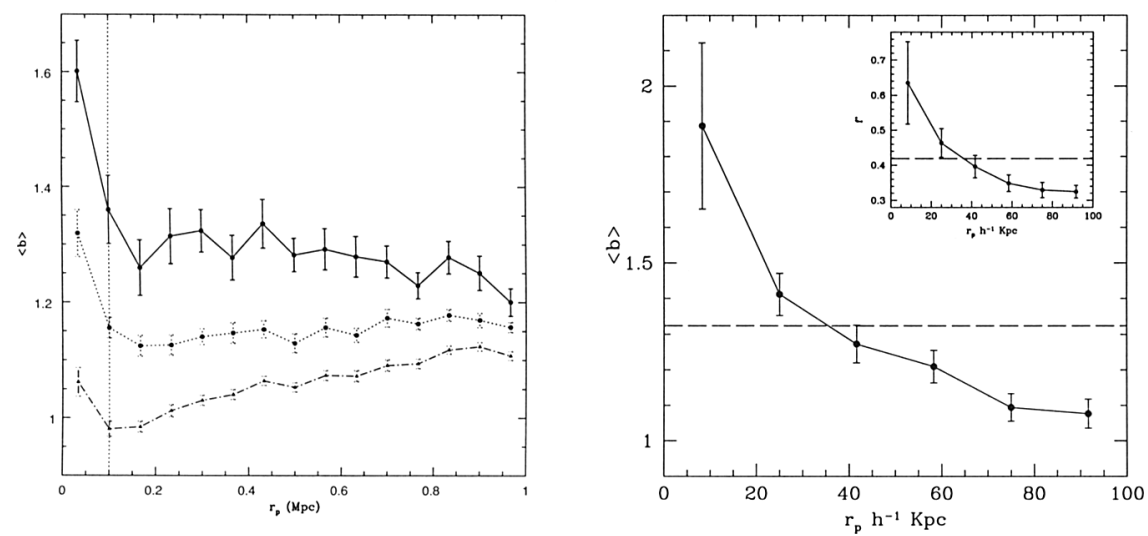

Figure 1. a) Mean birthrate parameter $b$ as a function of relative projected separation $r_{p}$ of galaxies with $\eta>3.5$ (solid line), $\eta>-1.4$ (dotted line) and no $\eta$ restriction (dotted-dashed line). The dotted vertical line depicts the spatial separation threshold identification. b) Mean $b$ parameters estimated in projected distance bins for galaxies in interacting pairs in the field. The small box shows the fraction $f^{\star}$ of galaxies with $b>\bar{b}$. The dashed horizontal lines represent the mean $b$ parameter for the corresponding control sample.

\section{References}

Alonso, M. S, Tissera, P. B., Coldwell, G., Lambas, D. G., 2003, submitted.

Barton E. J., Geller M. J., Kenyon S. J., 2000, ApJ, 530, 660

Lambas, D. G., Tissera, P. B., Alonso, M. S. Coldwell, G. 2003, MNRAS in press 